to identify desirable attributes of an inhaler. $(2,3)$ "Ease of use" is an important characteristic but is often determined after instructed training. Whilst the importance of initial and repeat correct inhaler technique training cannot be overlooked, a dry powder inhaler (DPI) device that is intuitively easy to use may be more beneficial for those patients with poor technique recall or with barriers to access adequate training. An intuitively easy to use inhaler such as the Sandoz Forspiro ${ }^{\mathrm{TM}}$ device may facilitate correct patient handling. A 28 day study to assess the intuitive ease of use of the Forspiro device was conducted in 24 Accuhaler ${ }^{\circledR}$ users ( $\geq 1$ year use), aged $20-86$ years (mean age $=53.8$ ). Participants were separated into 2 groups of 12 and received either limited written instructions about the use of the device (Uninstructed) or were given fully illustrated instructions for use (Instructed). See table 1 . The correlation of the ease of use assessment by Uninstructed and Instructed subjects has shown that the new Forspiro DPI is intuitive to use, even in patients that had received minimal instructions for use. The introduction of innovative generic versions of inhaled medications enables the much needed economic rationalisation of drug use in these times of austerity within the NHS. Benefitting from advanced design technology, drawn from years of patient feedback, generic versions of inhaled medications may be more readily adopted by patients and HCPs if the device is intuitive to use. The importance of initial and repeat correct inhaler technique training cannot be overlooked, however intuitively easy to use inhalers, such as the Forspiro device, may facilitate correct patient handling. Refs: 1. Giraud 2002; 2. Virchow 2008; 3. Chrystyn 2007

Abstract P7 Table 1 Subject ratings of ease of use of the novel Forspiro DPI device

\begin{tabular}{lcccc}
\hline & \multicolumn{2}{c}{ Instructed $(\mathbf{n}=12)$} & \multicolumn{2}{c}{ Uninstructed $(\mathbf{n = 1 2})$} \\
\hline & Very easy & Fairly easy & Very easy & Fairly easy \\
\hline Overall ease of use & 8 & 4 & $3^{*}$ & 8 \\
Ease of determining the number of & 10 & 2 & $7^{*}$ & 4 \\
doses left & 10 & 2 & 8 & 4 \\
Ease of preparing a dose & 8 & 4 & 8 & 4 \\
Ease of removing used blisters & & & & \\
\hline
\end{tabular}

*One subject in the uninstructed group rated this aspect "Fairly difficult".

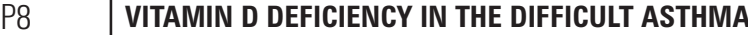 POPULATION; FINDINGS FROM A DIFFICULT ASTHMA CLINIC}

doi:10.1136/thoraxjnl-2012-202678.149

${ }^{1} \mathrm{VB}$ Joshi, 'B Dyke, ${ }^{1} \mathrm{~T}$ Rahman, ${ }^{2} \mathrm{C}$ Townshend, ${ }^{2} \mathrm{~V}$ Patil, ${ }^{1} \mathrm{R}$ Kurukullaratchy. ${ }^{1}$ Department of Respiratory Medicine, Mailpoint 52, Room CG-89, G-Level West Wing, Southampton General Hospital, Southampton, S016 6YD, UK; ${ }^{2}$ The David Hide Asthma and Allergy Research Centre, St Mary's Hospital, Isle of Wight, P030 5TG, UK

Introduction Recent interest has focussed on potential regulatory roles for vitamin $\mathrm{D}$ in the development and course of asthma.

Aim We assessed the prevalence and characteristics of patients with vitamin $\mathrm{D}$ deficiency, during a calendar year, among our Regional Difficult Asthma Clinic population.

Methods Retrospective study was undertaken to assess vitamin D deficiency prevalence plus characterisation of "deficient" compared to "normal" vitamin D level patients. Characterisation included demographic factors, associated comorbidities, asthma severity (BTS steps), and spirometry during that 12 month period. Comorbidities recorded included BodyMass Index (BMI), smoking status, atopic status, rhinitis, food allergy, salicylate sensitivity, sulphite sensitivity, Gastro-oesophageal Reflux disease (GORD), dysfunctional breathing, and psychological comorbidity.
Results Serum vitamin D3 assessment was available for $85.4 \%$ $(158 / 185)$ patients during the previous 12 months. First measured vitamin D3 levels were used for primary analysis showing a mean clinic vitamin D3 level of $45.1 \mathrm{nmol} / \mathrm{L}$. Normal vitamin D3 levels $(>72 \mathrm{nmol} / \mathrm{L})$ occurred in $18.9 \%, 14.6 \%$ had insufficient levels $(52-72 \mathrm{nmol} / \mathrm{L})$ and $65.5 \%$ had deficient levels $(<52 \mathrm{nmol} / \mathrm{L})$. Severe deficiency $(<20 \mathrm{nmol} / \mathrm{L})$ occurred in $14.6 \%$. Vitamin D deficiency showed highest prevalence during Winter, Autumn and Spring (83-79\%) and lowest level in Summer (53.3\%). Two thirds of patients received a course of vitamin $\mathrm{D}$ replacement therapy during the year.

Vitamin D deficient showed no difference compared to normal vitamin D3 status patients in atopic status, spirometry, inhaled medication usage, maintenance oral steroid use, Omalizumab use and asthma hospitalisations. No significant difference was observed between the two groups in respect to comorbidities like GORD, rhinitis, COPD, bronchiectasis, food allergy and dysfunctional breathing. However, vitamin $\mathrm{D}$ deficient subjects had significantly higher BMI (31.6 v $\left.26.4 \mathrm{~kg} / \mathrm{m}^{2} ; \mathrm{p}=0.007\right)$, obesity (BMI>30) $(55.3 \%$ v $13 \%$; $\mathrm{p}<0.001)$ and psychological comorbidity $(26.7 \%$ v $3.6 \% ; \mathrm{p}=0.009)$. Conclusion Vitamin D deficiency is highly prevalent in the Difficult Asthma group. The clinical relevance of this finding remains unclear. While vitamin $\mathrm{D}$ deficient patients did not show greater asthma treatment needs or healthcare use that might have reflected influence of receiving high dose vitamin D replacement. Patients with vitamin $\mathrm{D}$ deficiency showed significantly greater obesity and psychological comorbidity. The association of vitamin D and Difficult Asthma merits further attention.

\section{P9 ADRENAL SUPPRESSION IN DIFFICULT ASTHMA: A NEGLECTED CAUSE FOR CONCERN}

doi:10.1136/thoraxjnl-2012-202678.150

TJT Sutherland, C Hodgekiss, J Slough, JH Barth, IJ Clifton. St James' University Hospital, Leeds, United Kingdom

Introduction Inhaled corticosteroids (ICS) are the mainstay of treatment in asthma. Whilst they are felt to be safe in low doses, a number of case studies have demonstrated adrenal suppression in higher doses, particularly in paediatric patients. The relative frequency of this is largely unknown, but we believe it may be significantly underrecognised in patients with difficult to treat asthma. As part of our difficult asthma protocol we measure random cortisol levels and proceeded to further investigations if this was abnormal or the patient presented with symptoms of adrenal insufficiency.

Methods Patients were assessed through the Difficult Asthma Service in Leeds which is a tertiary referral centre for West Yorkshire. Patients who had non-specific symptoms out of keeping with asthma, either had a random serum cortisol taken or had a short synacthen test. Patients were classified as: adrenal insufficient with a cortisol less than $50 \mathrm{nmol} / \mathrm{L}$ or a positive short synacthen test, and adrenal sufficient with a random cortisol greater than $120 \mathrm{nmol} / \mathrm{L}$ or a negative short synacthen test. A further group was defined as suboptimal if their random cortisol was between 50-120nmol/L Data were also collected on ICS dose and type, and atopic status.

Results Ninety-two random cortisol samples have been taken to date. Of these, 8 patients who were not on oral corticosteroids were found to have adrenal insufficiency (8.7\%). Seven patients had a random cortisol less than $50 \mathrm{nmol} / \mathrm{L}$ and one had a positive short synacthen test. Of the patients with adrenal insufficiency six patients were on a combination inhaler including fluticasone, and all received a daily BDP dose over $1000 \mathrm{mcg}$. None of the patients with adrenal insufficiency demonstrated evidence of atopy, except one patient with co-existent ABPA receiving itraconazole therapy.

Conclusions We have identified a significant number of patients with evidence of adrenal insufficiency, with the majority identified by a random cortisol. This is likely to be under-estimated, as the 92 